\title{
Synthesis of cobalt nanowires in aqueous solution under an external magnetic field
}

\author{
Xiaoyu Li, Lijuan Sun, Hu Wang, Kenan Xie, Qin Long, Xuefei Lai and Li Liao*
}

\section{Letter}

\section{Address:}

School of Chemical Engineering, Sichuan University, Chengdu 610065, PR China

\section{Email:}

Li Liao* - liaolis@scu.edu.cn

* Corresponding author

Keywords:

aqueous solution; cobalt nanowires; external magnetic field; magnetic properties; surfactant
Beilstein J. Nanotechnol. 2016, 7, 990-994. doi:10.3762/bjnano.7.91

Received: 25 March 2016

Accepted: 24 June 2016

Published: 07 July 2016

This article is part of the Thematic Series "Physics, chemistry and biology of functional nanostructures III".

Guest Editor: A. S. Sidorenko

(c) 2016 Li et al.; licensee Beilstein-Institut. License and terms: see end of document.

\begin{abstract}
In contrast to the majority of related experiments, which are carried out in organic solvents at high temperatures and pressures, cobalt nanowires were synthesized by chemical reduction in aqueous solution with the assistance of polyvinylpyrrolidone (PVP) as surfactant under moderate conditions for the first time, while an external magnetic field of $40 \mathrm{mT}$ was applied. Uniform linear cobalt nanowires with relatively smooth surfaces and firm structure were obtained and possessed an average diameter of about $100 \mathrm{~nm}$ with a coating layer of PVP. By comparison, the external magnetic field and PVP were proven to have a crucial influence on the morphology and the size of the synthesized cobalt nanowires. The prepared cobalt nanowires are crystalline and mainly consist of cobalt as well as a small amount of platinum. Magnetic measurements showed that the resultant cobalt nanowires were ferromagnetic at room temperature. The saturation magnetization $\left(M_{\mathrm{s}}\right)$ and the coercivity $\left(H_{\mathrm{c}}\right)$ were $112.00 \mathrm{emu} / \mathrm{g}$ and $352.87 \mathrm{Oe}$, respectively.
\end{abstract}

\section{Findings}

In recent years, cobalt nanowires, as a ferromagnetic material, have attracted considerable attention due to their outstanding magnetic properties and excellent performance in applications in high-density magnetic storage media [1,2], in immune magnetic separation [3], in gene delivery [4] and as targeted drug carrier [5]. Hydrothermal and solvothermal methods are welldeveloped approaches to fabricate cobalt nanowires [6-10]. However, such methods set high requirements for the equip-

ment because of high temperatures and pressures. Therefore, researches concerning a solution-reduction method under moderate conditions for preparing cobalt nanowires have been carried out due to their simplicity and low cost $[11,12]$. Organics, such as ethylene glycol and propylene glycol, are used to serve as solvents in most of the experiments. They can provide stable process for preparing nanowires owing to the relatively high viscosity of these solvents. Reactions proceed 
gently in organic solvents, which results in a high degree of crystallization and a high dispersibility of the nanowires. Nevertheless, organic solvents are not easily washed out of products, and the most important problem is that they are not environmentally friendly. Compared to organic solvents, the use of water as the solvent is cheaper and more convenient and does less harm to the environment. There are few reports on preparing cobalt nanowires in aqueous solution up to now [13]. In our previous studies, we have successfully prepared nickel and nickel/copper nanowires [14-16], and cobalt nanowires were synthesized using $\mathrm{NaBH}_{4}$ as initiator without surfactant in ethylene glycol solution with an external magnetic field under moderate conditions [12]. In this research, with polyvinylpyrrolidone (PVP) as surfactant, uniform linear cobalt nanowires with a mean diameter of about $100 \mathrm{~nm}$ were synthesized in aqueous solution under an external magnetic field at low temperature and atmosphere pressure for the first time.

All the chemical reagents used in the experiment were analytical grade. $\mathrm{CoCl}_{2} \cdot 6 \mathrm{H}_{2} \mathrm{O}$, EDTA-2Na and PVP acted as Co precursor, chelating agent and surfactant, respectively. $\mathrm{N}_{2} \mathrm{H}_{4} \cdot \mathrm{H}_{2} \mathrm{O}$ and $\mathrm{H}_{2} \mathrm{PtCl}_{6} \cdot 6 \mathrm{H}_{2} \mathrm{O}$ worked as reductant and initiator, respectively. The synthesis was performed inside water bath set at $80{ }^{\circ} \mathrm{C}$. Firstly, $6 \mathrm{mmol} \mathrm{CoCl} 2 \cdot 6 \mathrm{H}_{2} \mathrm{O}$ and 6 mmol EDTA-2Na were dissolved in $60 \mathrm{~mL}$ distilled water inside a polytetrafluoroethylene beaker placed between two magnets, with an applied field of $40 \mathrm{mT}$ measured by a HT20 tesla meter. Then $\mathrm{NaOH}$ was added to adjust the $\mathrm{pH}$ value of the solution to about 14 , and $0.18 \mathrm{~g}$ PVP was dissolved in the solution by thorough stirring. Next, $0.30 \mathrm{~mL} \mathrm{~N} \mathrm{H}_{4} \cdot \mathrm{H}_{2} \mathrm{O}$ and $0.12 \mathrm{~mL}$ of $0.0253 \mathrm{M}$ $\mathrm{H}_{2} \mathrm{PtCl}_{6} \cdot 6 \mathrm{H}_{2} \mathrm{O}$ were mixed with the solution. The reaction proceeded for $15 \mathrm{~min}$, during which nanowires were formed gradually. Finally, the prepared nanowires were separated from the solution with a magnet, and washed ultrasonically in distilled water and ethanol three times each to remove the organics from the surface of the nanowires.

The size and morphology of the synthesized nanowires were observed with a field emission scanning electron microscope (SEM, JEOL JSM-7500F) and a transmission electron microscope (TEM, JEOL JEM-100CX). The composition and crystal structure of the freeze-dried products were characterized by energy dispersive spectrometry (EDS, Oxford Instruments X-Max 51-XMX0019), X-ray diffraction (XRD, Philips X'Pert Pro MPD) in the range from $20-90^{\circ}$ using $\mathrm{Cu}$ radiation $(\lambda=0.154249 \mathrm{~nm})$ with an incremental step size of $4 \% \mathrm{~min}$, the generator voltage of $40 \mathrm{kV}$ and tube current of $40 \mathrm{~mA}$, and selected area electron diffraction (SAED, FEI Tecnai-G20). The magnetic properties, such as saturation magnetization $\left(M_{\mathrm{S}}\right)$ and coercivity $\left(H_{\mathrm{c}}\right)$, were studied by a magnetic property measurement system superconducting quantum interference device
(Quantum Design, Inc., MPMS SQUID XL) at room temperature using an applied field of up to $2.5 \mathrm{~T}$.

Figure 1a,b show SEM images of cobalt nanowires prepared with PVP in aqueous solution under an external magnetic field. Uniform linear cobalt nanowires with a mean diameter of about $100 \mathrm{~nm}$ were observed, which were much smaller than those prepared in our previous studies in diameter (about $500 \mathrm{~nm}$ ) [12]. The cobalt nanowires possessed relative smooth surface without apparent aggregation. Figure 1c shows the TEM image of the PVP-protected cobalt nanowires prepared under an external magnetic field. The nanowires exhibited a firm linear structure without any gap. Furthermore, the diameter of the nanowire shown in the TEM image is about $60 \mathrm{~nm}$, while that in Figure $1 \mathrm{~b}$ is about $100 \mathrm{~nm}$. The obvious difference in diameter observed by SEM and TEM indicated that synthesized cobalt nanowires were uniformly coated by a layer of PVP whose thickness was about $20 \mathrm{~nm}$. Figure $1 \mathrm{~d}-\mathrm{f}$ shows SEM and TEM images of cobalt nanowires prepared without PVP in aqueous solution under an external magnetic field. In the absence of PVP, nanowires with a relatively rough surface were obtained, whose size distribution was in the range of 200 to $350 \mathrm{~nm}$. There were also some chain-like structures observed by SEM. It demonstrated that PVP, as a surfactant, had a crucial influence on the morphology and the size of cobalt nanowires

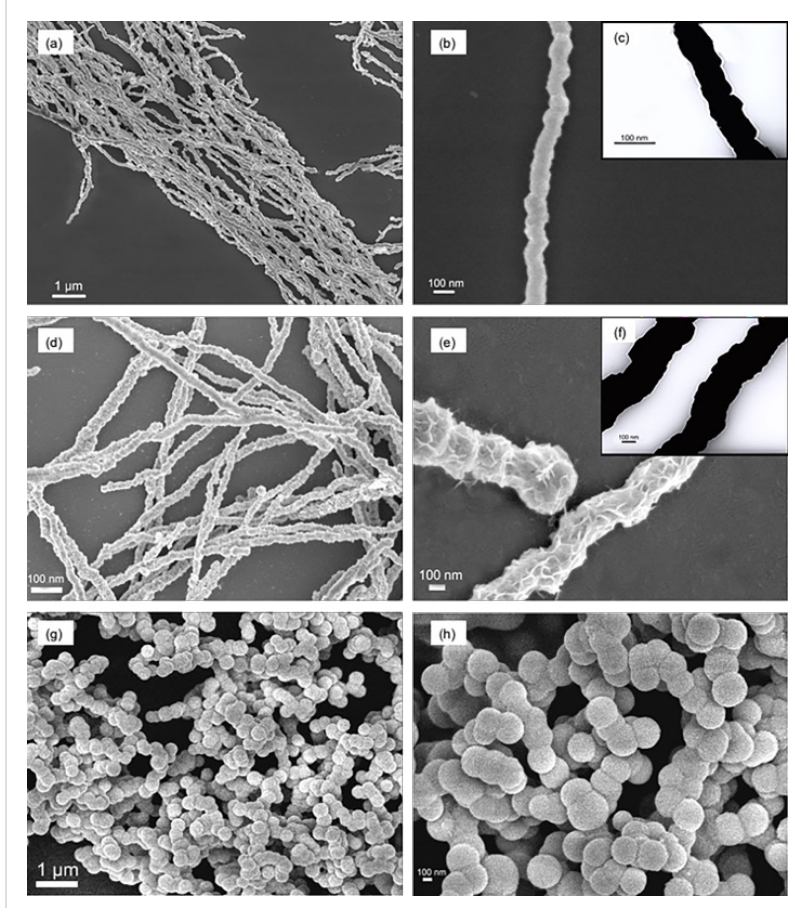

Figure 1: SEM images of cobalt nanowires prepared under an external magnetic field with PVP $(a, b)$ and without PVP $(d, e)$, and cobalt nanowires prepared without an external magnetic field $(\mathrm{g}, \mathrm{h})$. The insets (c, f) show the corresponding TEM images of cobalt nanowires $(b, e)$, respectively. 
synthesized via this method. Figure 1g,h shows SEM images of cobalt nanowires prepared without an external magnetic field. In the absence of an external magnetic field, only spherical particles with the diameter of about $250 \mathrm{~nm}$ were obtained, which aggregated without an obvious orientation. Comparing the diameter with that of the nanowires in Figure $1 \mathrm{~b}$, it can be inferred that applying an external magnetic field could inhibit the growth of the products. During the experimental process, small cobalt nanoparticles were generated in the solution at first, regardless of the presence of the external magnetic field. Then these small nanoparticles agglomerated and formed larger particles that started to align along the external magnetic field. As the reaction proceeded, Co(II) ions in the solution were reduced and deposited in the gaps between particles, resulting in the formation of cobalt nanowires $[11,14]$.

Figure 2 shows the EDS spectrum of the PVP-protected cobalt nanowires prepared under an external magnetic field. Weight percent (wt \%) and atom percent (atom \%) of each element were determined by EDS analysis. The silicon peak stems from the silicon wafer substrate. For the sake of clarity it was left out from the calculation of the elemental composition. Cobalt and platinum acting as heterogeneous nucleation sites are clearly seen. Carbon and oxygen can be ascribed to the PVP coating on the nanowires and, to some extent, to $\mathrm{CO}_{2}$ adsorbed on nanowires during the preparation of samples. There is also very little residual chlorine detected, which comes from $\mathrm{H}_{2} \mathrm{PtCl}_{6} \cdot 6 \mathrm{H}_{2} \mathrm{O}$.

XRD patterns of the cobalt nanowires prepared with PVP and without PVP under an external magnetic field are shown in Figure 3a,b, respectively. The corresponding SAED patterns are shown in Figure 3c,d. The diffraction peaks of cobalt nano-

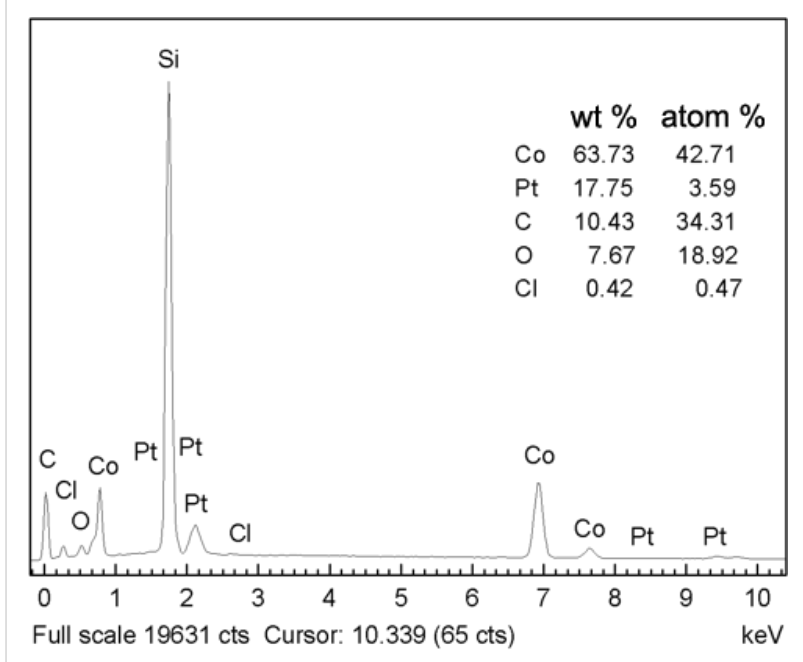

Figure 2: EDS spectrum of the PVP-protected cobalt nanowires prepared under an external magnetic field. Silicon was left out from the composition evaluation for the sake of clarity.

wires prepared with PVP and without PVP could be indexed with the reflections of face-centered cubic (fcc) Co (PDF standard cards, JCPDS 15-0806, space group $F m-3 m$ ). Two peaks of fcc $\operatorname{Co}\left(2 \theta=44.43^{\circ}\right.$ and $\left.2 \theta=75.94^{\circ}\right)$ corresponding to Miller indices (111) and (220), respectively, were observed in each XRD pattern, and a diffraction ring as well as some scattered diffraction mottling were shown in each SAED pattern, which demonstrated that the resultant nanowires possessed crystal structure and PVP had only little impact on that.

Figure 4 displays the hysteresis loop measured at room temperature under an applied magnetic field of up to 25000 Oe for the PVP-protected cobalt nanowires obtained in aqueous solution
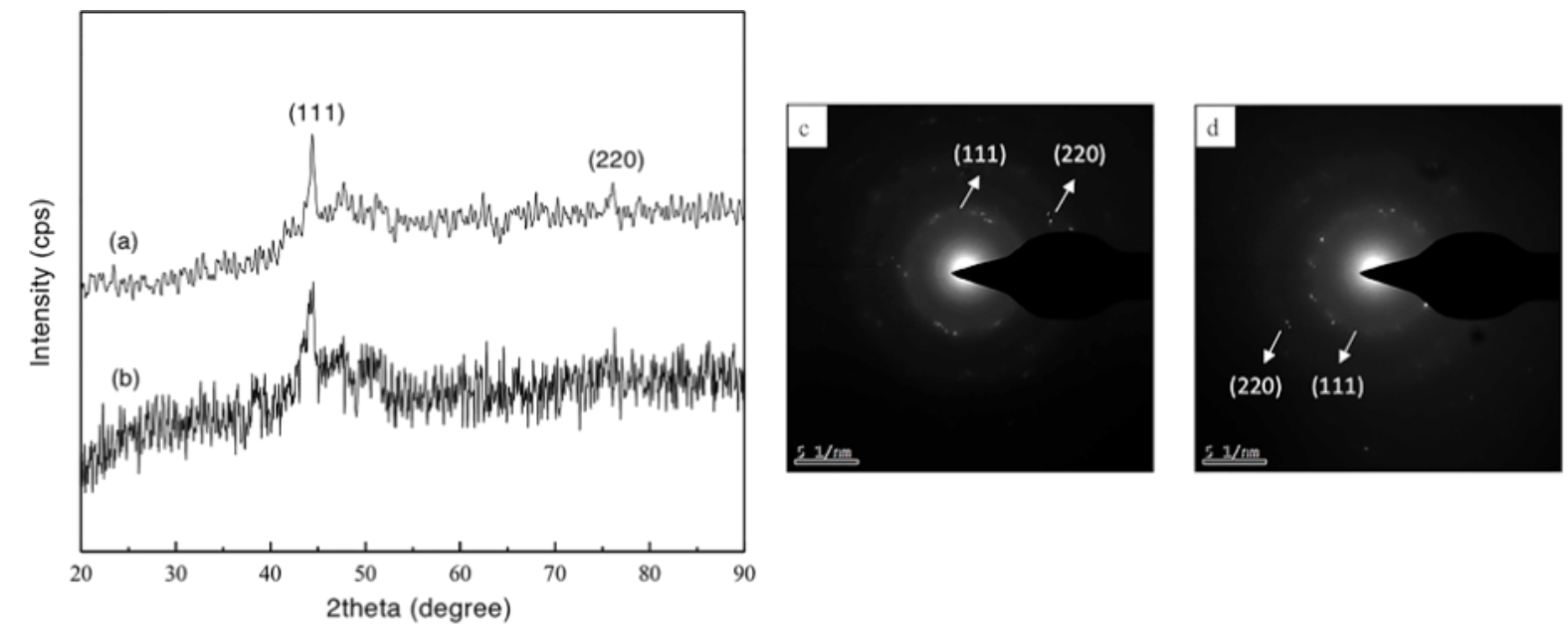

Figure 3: XRD patterns of cobalt nanowires prepared with PVP (a) and without PVP (b) under an external magnetic field. The corresponding SAED patterns of cobalt nanowires prepared with PVP (c) and without PVP (d). 


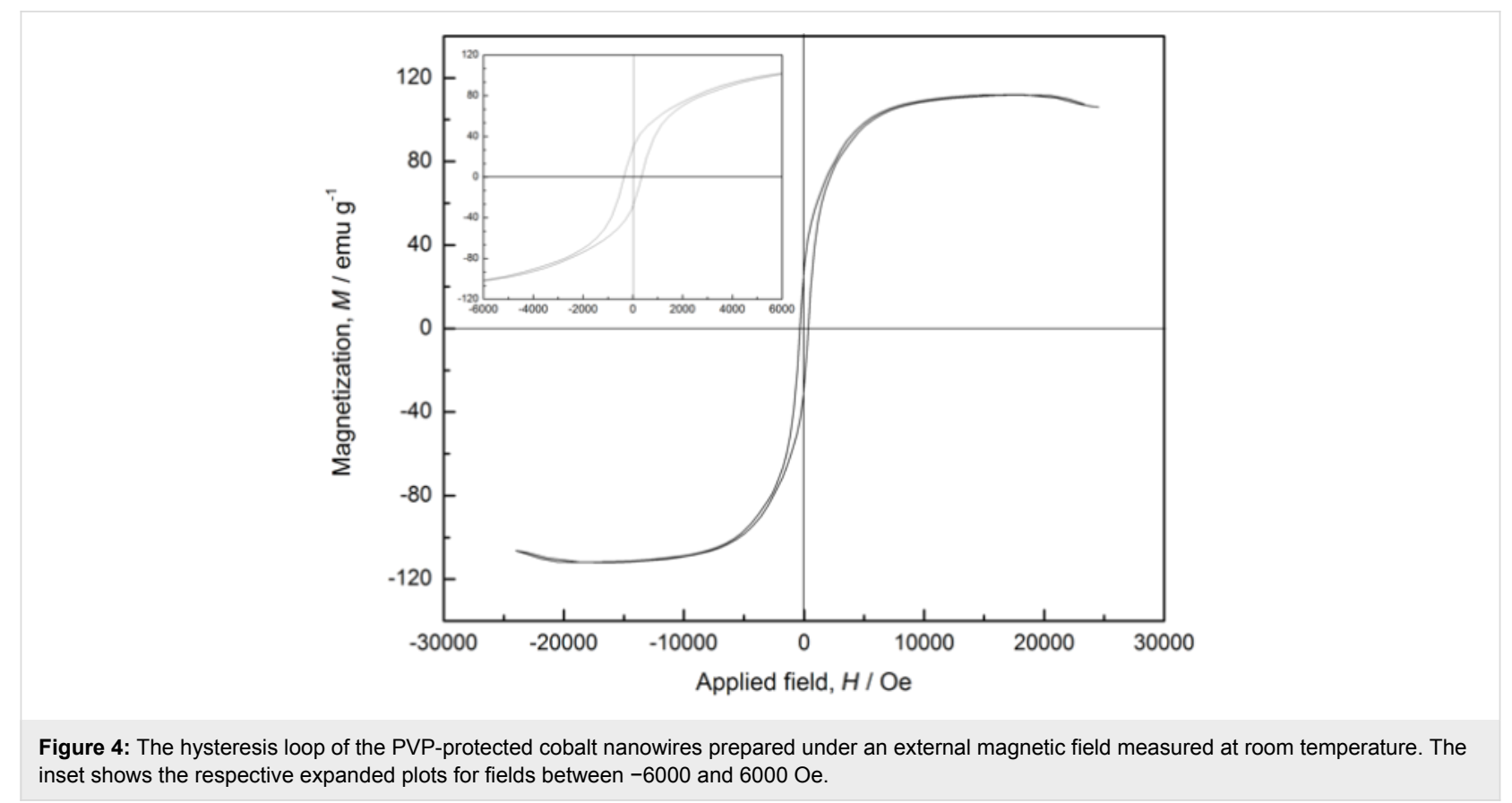

under an external magnetic field of $40 \mathrm{mT}$. An expanded plot is shown in the insert for field strengths between -6000 Oe and 6000 Oe. The hysteresis loop suggested that the synthesized cobalt nanowires were ferromagnetic at room temperature, which differs from the superparamagnetic property exhibited in the previous report [13]. The coercivity $\left(H_{\mathrm{c}}\right)$ of the cobalt nanowires were $352.87 \mathrm{Oe}$, and the saturation magnetization $\left(M_{\mathrm{s}}\right)$ were $112.00 \mathrm{emu} \mathrm{g}^{-1}$, which was lower than the corresponding value of bulk cobalt (162.5 emu $\left.\mathrm{g}^{-1}\right)$ [11].

In summary, uniform linear cobalt nanowires with a mean diameter of about $100 \mathrm{~nm}$ were obtained by chemical reduction in aqueous solution with an external magnetic field for the first time. The cobalt nanowires exhibited a relatively smooth surface and firm structure with a layer of PVP, which had a significant impact on the morphology and size of nanowires. The synthesized nanowires mainly consisted of cobalt and a small amount of platinum with crystal structure. Cobalt nanowires prepared were ferromagnetic at room temperature. The saturation magnetization $\left(M_{\mathrm{s}}\right)$ and the coercivity $\left(H_{\mathrm{c}}\right)$ were $112.00 \mathrm{emu} / \mathrm{g}$ and $352.87 \mathrm{Oe}$, respectively.

\section{Acknowledgements}

Financial supports by the National Natural Science Foundation of China (Grant No. 50904046) is gratefully acknowledged.

\section{References}

1. Saini, D.; Chauhan, R. P.; Kumar, S. J. Mater. Sci.: Mater. Electron. 2013, 25, 124-127. doi:10.1007/s10854-013-1560-0
2. Liakakos, N.; Blon, T.; Achkar, C.; Vilar, V.; Cormary, B.; Tan, R. P.; Benamara, O.; Chaboussant, G.; Ott, F.; Warot-Fonrose, B.; Snoeck, E.; Chaudret, B.; Soulantica, K.; Respaud, M. Nano Lett. 2014, 14, 3481-3486. doi:10.1021/nl501018z

3. Hultgren, A.; Tanase, M.; Chen, C. S.; Reich, D. H. IEEE Trans. Magn. 2004, 40, 2988-2990. doi:10.1109/TMAG.2004.830406

4. Raphael, M. P.; Christodoulides, J. A.; Qadri, S. N.; Simpkins, B. S.; Byers, J. M. Nanotechnology 2010, 21, 285101. doi:10.1088/0957-4484/21/28/285101

5. Gao, W.; Wang, J. Nanoscale 2014, 6, 10486-10494. doi:10.1039/C4NR03124E

6. Niu, H.; Chen, Q.; Zhu, H.; Lin, Y.; Zhang, X. J. Mater. Chem. 2003, 13 1803-1805. doi:10.1039/b303024e

7. Xie, B.-Q.; Qian, Y.; Zhang, S.; Fu, S.; Yu, W. Eur. J. Inorg. Chem. 2006, 2006, 2454-2459. doi:10.1002/ejic.200600061

8. Wang, M.; Xiong, J.; Sun, Y.; Chen, Q. CrystEngComm 2010, 12, 3262-3266. doi:10.1039/c000439a

9. Dong, X.; Qi, M.; Tong, Y.; Ye, F. Mater. Lett. 2014, 128, 39-41. doi:10.1016/j.matlet.2014.04.133

10. Gandha, K.; Elkins, K.; Poudyal, N.; Liu, X.; Liu, J. P. Sci. Rep. 2014, 4, 5345. doi:10.1038/srep05345

11. Balela, M. D. L.; Yagi, S.; Matsubara, E. J. Electrochem. Soc. 2011, 158, D210-D216. doi:10.1149/1.3545065

12. Li, M.; Xie, K.; Wu, Y.; Yang, Q.; Liao, L. Mater. Lett. 2013, 111, 185-187. doi:10.1016/j.matlet.2013.08.088

13. Xiong, Y.; Chen, Q.; Tao, N.; Ye, J.; Tang, Y.; Feng, J.; Gu, X. Nanotechnology 2007, 18, 345301. doi:10.1088/0957-4484/18/34/345301

14. Li, X.; Wang, H.; Xie, K.; Long, Q.; Lai, X.; Liao, L. Beilstein J. Nanotechnol. 2015, 6, 2123-2128. doi:10.3762/bjnano.6.217

15. Wang, H.; Li, M.; Li, X.; Xie, K.; Liao, L. Bull. Mater. Sci. 2015, 38 , 1285-1289. doi:10.1007/s12034-015-1012-y

16. Wang, H.; Li, X.; Li, M.; Xie, K.; Liao, L. Beilstein J. Nanotechnol. 2015, 6, 1268-1271. doi:10.3762/bjnano.6.130 


\section{License and Terms}

This is an Open Access article under the terms of the Creative Commons Attribution License

(http://creativecommons.org/licenses/by/2.0), which permits unrestricted use, distribution, and reproduction in any medium, provided the original work is properly cited.

The license is subject to the Beilstein Journal of Nanotechnology terms and conditions:

(http://www.beilstein-journals.org/bjnano)

The definitive version of this article is the electronic one which can be found at:

doi:10.3762/bjnano.7.91 